\title{
Can streamer blobs prevent the buildup of the interplanetary magnetic field?
}

\author{
M. K. van Aalst \\ Sterrekundig Instituut, Universiteit Utrecht, Postbus 80 000, 3508 TA Utrecht, Netherlands; \\ aalst@phys.uu.nl \\ P. C. H. Martens", A. J. C. Beliën \\ Solar System Division, ESA Space Science Department at Goddard Space Flight Center, Greenbelt, 20771, \\ MD, U.S.A.; pmartens@solar.stanford.edu, sbelien@esa.nascom.nasa.gov
}

\begin{abstract}
Coronal Mass Ejections continuously drag closed magnetic field lines away from the Sun, adding new flux to the interplanetary magnetic field (IMF). We propose that the outwardmoving blobs that have been observed in helmet streamers are evidence of ongoing, small-scale reconnection in streamer current sheets, which may play an important role in the prevention of an indefinite buildup of the IMF. Reconnection between two open field lines from both sides of a streamer current sheet creates a new closed field line, which becomes part of the helmet, and a disconnected field line, which moves outward. The blobs are formed by plasma from the streamer that is swept up in the trough of the outward moving field line. We show that this mechanism is supported by observations from SOHO/LASCO. Additionally, we propose a thorough statistical study to quantify the contribution of blob formation to the reduction of the IMF, and indicate how this mechanism may be verified by observations with SOHO/UVCS and the proposed NASA STEREO and ESA Polar Orbiter missions.
\end{abstract}

Subject headings: interplanetary medium, solar wind, Sun: corona, Sun: magnetic fields, Sun: particle emission

\section{Introduction}

Using the LASCO C2 and C3 coronagraphs on the SOHO spacecraft (Brueckner et al. 1995), Sheeley et al. (1997) discovered blobs of material moving outward in coronal helmet streamers. Helmet streamers consist of a bubble-like or arch-like wide body (the helmet), consisting of closed magnetic field. Above the cusp, the pointed top of the helmet, lies a current sheet which extends radially outward, separating the two directions of open magnetic field around the helmet (see Fig. 1, top panel). We adopt the view that this streamer structure is formed by reconnection of open field lines in the current sheet that is left behind by a CME, as suggested by, e.g., Kopp (1992) and Kahler \& Hundhausen (1992). Sheeley et al. (1997) observed that the blobs originate in the streamer current sheet, right above the cusp. They have an initial size of about $1 R_{\odot}$ in the radial and $0.1 R_{\odot}$ in the transverse direction. The blobs move radially outward along the streamer, with increasing velocities from about $150 \mathrm{kms}^{-1}$ near $5 R_{\odot}$ to $300 \mathrm{kms}^{-1}$ near $25 R_{\odot}$. Because of their relatively small initial sizes, low intensities $(\Delta I / I \lesssim 0.1)$, radial motions, slow but increasing velocities, and location in the streamer belt, Sheeley et al. (1997) conclude that these features passively trace the solar

\footnotetext{
${ }^{1}$ As of January 1998: Department of Physics, Montana State University, P.O. Box 173840, Bozeman, MT 59717-3840 U.S.A.
} 
wind. Wang et al. (1998) carried out some more detailed observations of the spatial distribution, relative intensities, and shapes of the blobs, and observed a rather steady occurrence rate of about four blobs per day. We believe that the creation of these blobs is connected to a longstanding problem in solar physics: maintaining a roughly constant amount of flux in the interplanetary magnetic field (IMF).

Coronal Mass Ejections (CMEs) generally originate in regions of closed magnetic field. These field lines are torn away from the Sun and stretched out into distended loops that extend well into interplanetary space (Hundhausen 1997). In principle, each consecutive CME thus introduces new flux into the heliosphere. This would result in an indefinite growth of the IMF, which is not observed (e.g. Gold 1962; Gosling 1975; MacQueen 1980; McComas, Gosling, \& Phillips 1992). Apparently, some kind of reconnection takes place on the field lines that are torn away from the Sun, disconnecting the tops of the loops and returning new closed field lines to the Sun.

The reconnection must start shortly after the CME's departure, but it cannot be restricted to that first period. In situ measurements near and beyond 1 AU (e.g. Gosling, Birn, \& Hesse 1995; Gosling 1996) show that most field lines within CMEs are still connected to the Sun at both ends. However, a few field lines are connected on only one end, while others are completely disconnected. Additionally, about one third of the CMEs exhibit a flux-rope topology, characterized by a series of helical field lines wrapped around a central axis. Both this helicity and the disconnected field lines can only be explained by magnetic reconnection behind the CME during the days before it reaches 1 AU (e.g. Gosling et al. 1995). On the other hand, to prevent an indefinite buildup of the IMF by field lines that are still connected to the Sun, reconnection must also keep taking place after the CME has passed 1 AU, several days after it has left the Sun.

Three types of events show evidence for reconnection directly behind a departing CME. First, about one third of all CMEs are accompanied by long-duration (many hours) X-ray emission from expanding loops or arcades of loops (see Gosling 1993). Non-thermal emission at X-ray and radio wavelengths is often observed in conjunction with these events (Webb \& Cliver 1995). A beautiful example of a reforming helmet streamer is presented by Hiei, Hundhausen, \& Sime (1993). All these observations confirm the model of Kopp \& Pneuman (1976) which explains hot loop formation by magnetic reconnection behind the CME. Second, there are some observations of moving metric type IV radio events which are interpreted as emission from electrons that have become trapped in disconnected plasmoids within CMEs (e.g. Kundu et al. 1989). Third, there are many coronagraph observations of large circular, ovoidal or outward U- or V-shaped structures that are usually interpreted as disconnected CMEs (e.g. Illing and Hundhausen 1983). Webb \& Cliver (1995), analyzing all space-borne coronagraph and eclipse data up to then, concluded that possibly over $10 \%$ of all CMEs fall in this category. Unfortunately, due to their infrequency compared to CMEs, even these three mechanisms combined cannot explain the necessary amount of flux-disconnection.

An even bigger problem is posed by reconnection long after the CME. There have been suggestions that such reconnection would occur in interplanetary space itself (e.g. Wilcox 1971), but the new closed loops that would have to return to the Sun have not been observed (Gosling 1975; McComas et al. 1989). McComas et al. $(1989,1991)$ suggested that the large U-shaped disconnections described above are not related to a departing CME, but occur all by themselves across the heliospheric current sheet. However, triggering these large reconnections by emerging flux elsewhere in the corona seems rather ad-hoc (Webb \& Cliver 1995). Additionally, these events are still quite infrequent compared to CMEs. Evidently, the mechanism, or combination of mechanisms, that can disconnect enough flux to offset the constant flow of CMEs has not yet been identified. 
In this Letter, we suggest that the creation of blobs in coronal streamers is a mechanism for ongoing, small-scale reconnection that can disconnect open field lines and reform closed magnetic loops on the Sun. Especially since the blobs occur not just shortly after CMEs, but also rather steadily under quiet coronal conditions, they may play an important role in maintaining the roughly constant IMF.

\section{Mechanism}

We suggest that the blobs are the result of reconnection between two open field lines from both sides of a streamer current sheet. Naturally, we speak about field lines only to visualize what is going on; in reality, a finite amount of magnetic field will be involved in such a reconnection event. The field line topologies before and after the reconnection are outlined in Fig. 1. Reconnection between the two innermost open field lines in the top panel creates two new field lines. The first, with both ends connected to the Sun, is a new closed loop which becomes part of the helmet (as in e.g. Kahler \& Hundhausen 1992). The other one, with its ends extending into interplanetary space, will move outward. The blobs are formed by plasma from the streamer that is swept up in the trough of this outward moving field line (middle and bottom panels). Considering the exponential decay of the density in the streamer with radial distance, almost all of the material in the blob is collected right away, just above the cusp.

The loop that is moving outward will at first attempt to do so at the Alfvén speed. However, it is immediately slowed down when it sweeps up the plasma on its way. Nevertheless, the blob of material in the field line will accelerate faster than the surrounding wind, at least until the Lorentz force is balanced by the pressure differential that is built up when the plasma is swept up into a blob or, alternatively, until $\beta \gtrsim 1$ and the surrounding gas pressure starts to dominate the magnetic field. From then on, the field lines, and therefore the blobs, more or less flow along with their surroundings, tracing, as Sheeley et al. (1997) suggested, the slow solar wind.

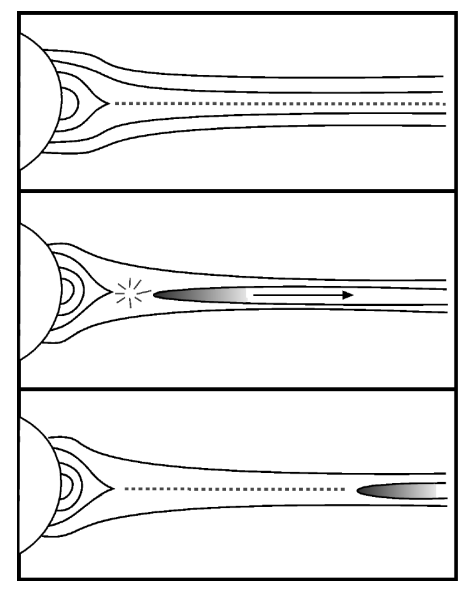

Fig. 1. The formation of a blob. The top panel shows the initial situation: a coronal streamer consisting of a helmet of closed loops surrounded by open field lines separated by a current sheet. In the lower two panels, reconnection has taken place between the two innermost field lines from the top panel. One new field line is a closed loop which becomes part of the helmet. The other one is now disconnected and moves outward, as shown in the bottom panels. On its way, it sweeps up plasma from the current sheet, which forms a blob. 
Einaudi et al. (1998) and Dahlburg, Boncinelli, \& Einaudi (1998) performed numerical simulations of a current sheet, suggesting that the fast solar wind flowing along a current sheet can trigger, amongst others, a resistive instability, resulting in plasmoid formation. The initial situation in these simulations was a one-dimensional equilibrium, a plane current sheet, which is then destabilized by two-dimensional disturbances. The model does not incorporate the helmet and the beginning of the current sheet, i.e. the cusp. We believe, however, that reconnection will occur preferentially just there. First, by virtue of the magnetic topology, the cusp is the most natural location for reconnection. Second, we notice that when plasma flows outward along the open field lines on the side of the helmet, an inward "inertial pressure" will arise at the cusp, where the plasma has to change direction to follow the field lines, which bend to become aligned with the streamer stalk. This pressure pushes the open field lines a little closer together right above the cusp. The reconnection that follows produces the new loops, as described above. Further study of both the location of reconnection and the subsequent movement of the blobs is needed. Numerical simulations would have to include, amongst others, the complete magnetic topology, the radially decreasing background density, and the acceleration of the surrounding solar wind.

\section{Evidence}

Several features of the blobs support the proposed mechanism. The first observation is the acceleration pattern of the blobs. Sheeley et al. (1997) observed that, in general, the blobs exhibit a fairly constant acceleration. However, in a speed-height plot representing measurements of about 65 independent blobs, they observed a peculiar "corner" at about 6 or $7 R_{\odot}$. Although this corner could be an artifact, Sheeley et al. (1997) remark that it might be a valid indication of a somewhat steeper initial acceleration. We suggest that the outward magnetic forces of the newly formed field line which sweeps up the plasma could account for this extra acceleration. As soon as $\beta>1$ and the magnetic field no longer dominates the gas pressure, the field line just moves along with the slow solar wind, as Sheeley et al. (1997) suggested.

Second, the blobs often exhibit a concave-outward V-shape when the plasma sheet in which they move outward is slightly inclined to the line of sight (Wang et al. 1998). This supports the idea that a field line, with a V-(or U-)shaped trough at its bottom, is sweeping up plasma. However, the fact that the V-shape only becomes visible when the sheet is seen at an angle calls for a further explanation, which is illustrated in Fig. 2. This figure represents the blob creation, but now seen both from our viewpoint, in the plane of the sky (bottom row) and from the solar pole, in the plane of the current sheet (top row). The two field lines shown in the top left panel correspond to the innermost open field lines in the bottom left panel. The outgoing magnetic flux rope has little room to expand in the direction perpendicular to the current sheet, which, by its very nature, is very thin and bounded by magnetic fields. Thus, when the current sheet is seen side-on, the swept-up plasma looks like a narrow blob, as shown in the bottom right panel. However, there probably is a substantial azimuthal component to the magnetic field of the streamer (top left panel), e.g. due to differential rotation. This leads to a V-shape of the newly reconnected field line when seen from above. Consequently, from this point of view, the plasma that is swept up will be V-shaped as well (top right panel). As long as the current sheet is perpendicular to the plane of the sky, this does not affect our image of the blob; we simply see a projection that is still shaped like an elongated blob (as in the bottom right panel). However, when the sheet is inclined to the line of sight, the V-shape will become visible. We

notice that an ESA Polar Orbiter at about 0.5 AU, a candidate mission for around 2007 (Priest et al. 1998), could observe the structures drawn in the top panels of Fig. 2. 


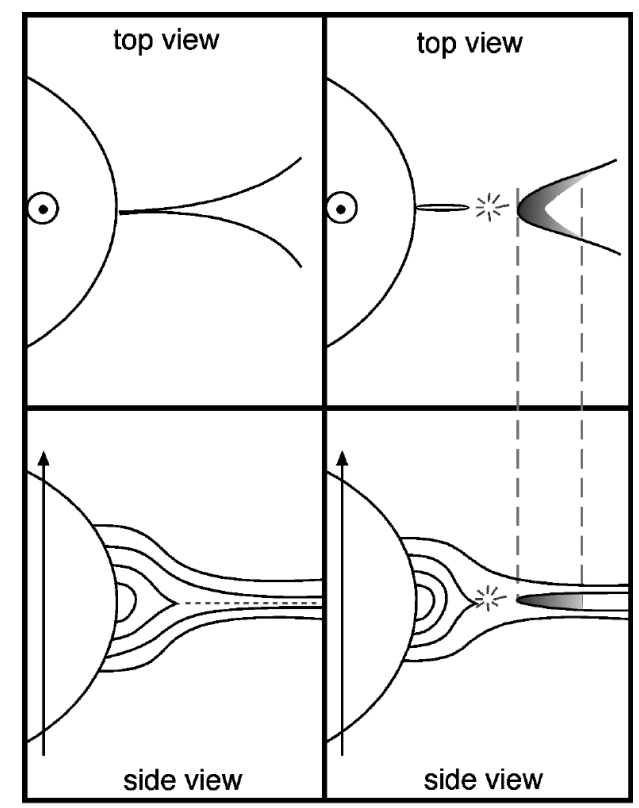

Fig. 2. The formation of a blob, seen from two directions. The bottom panels show the streamer in the plane of the sky, as we see it. The top panels show the same picture, but now seen from above. The two field lines in the top left panel correspond to the innermost open field lines in the bottom left panel. The two left panels show the situation before, the two right panels after the reconnection.

Third, it should be noted that the blobs remain coherent all through the LASCO-coronagraph's field of view, out to $30 R_{\odot}$. According to Sheeley et al. (1997), they maintain constant angular spans and increase their lengths in rough accord with their speeds. This appearance is consistent with our model of a density enhancement that is bound on both sides by magnetic field lines, which maintain a roughly constant angular span when they extend more or less radially from the Sun (in the plane of the sky), and from below by the magnetic trough which initially sweeps up the material in the blob.

Fourth, the blobs are not very bright, but do contain quite some material right from the moment they appear above the helmet. Sweeping up the material in a field line that is moving outward in an exponentially decreasing density is a very effective way to accomplish that. Another way would be to collect the material in a closed field line in the helmet. Considering that there is no observational evidence for the complete eruption of such a closed field line, Wang et al. (1998) have suggested that the material is released along an open field line after a reconnection between a closed field line and a nearby open one. However, such a mechanism makes it much harder to explain the three observations mentioned above. Recent measurements of abundances in streamers by Raymond et al. (1997) with the UVCS provide a definite test for whether the blob material comes out of the closed helmet or, as we propose, from the current sheet. These measurements show that elements with a high first ionization potential, like oxygen, are underabundant by an order of magnitude in the closed helmet, but only by a factor three on the sides of the helmet, where the field lines are open. Measurements of the abundances in blobs would thus allow us to determine whether their plasma originates in the closed helmet or in the open field lines adjoining the current sheet. In situ measurements of the slow solar wind provide another test: if the blobs are formed in the helmet instead of above it, the slow solar wind should contain a component exhibiting the specific characteristics of that material (Wang et al. 1998). 
Fifth, Wang et al. (1998) observed that the creation of the blobs is sometimes accompanied by a downflow of material. They compare this with downflows in the aftermath of CMEs, accompanying the closing down of fields blown open during the event. Clearly, this fits our model very well. While the outward moving loop will usually be most visible, the new closed loop will also collect some material while it is moving downward to become part of the helmet.

Finally, we suggest that additional observational evidence for this mechanism can be collected by looking at the development of a helmet after the departure of a blob. The reconnection between open field lines not only releases a field line, which then forms a blob, but also adds a new closed field line to the helmet, which should grow in size (with a little delay to allow it to be filled up with material from below). We notice that a measurement of this growth, combined with an estimate of the field strength at the bottom of the helmet, immediately yields the flux involved in the reconnection. Unfortunately, the solar rotation complicates this otherwise straightforward observational test. The additional field lines would presumably add at most a few percent to the size of the streamer. In coronagraph observations, such changes may just as well be the result of projection effects of structures that rotate into (or out of) view, aggravated by the contribution function for the visibility of material out of the plane of the sky (Hundhausen 1993). However, this problem can be overcome with a thorough statistical study of the relation between the expulsion of the blobs and the size and intensity of the helmet in a large number of streamers. We have not yet performed such a study, but preliminary observations of a smaller number of streamers yielded promising results. As

an alternative to statistical studies, some of the problems of the solar rotation could also be overcome with the proposed NASA STEREO mission, to be flown from mid-2003 (Rust 1997), which would observe the Sun from two different angles.

\section{Summary}

We have suggested that the origin of the blobs in coronal streamers that were observed by Sheeley et al. (1997) is related to the longstanding problem of maintaining a roughly constant flux in the interplanetary magnetic field. Two open field lines, from both sides of the current sheet, reconnect to form two new loops. One is a closed field line and becomes part of the helmet. The other is now disconnected from the Sun and moves outward, sweeping up the material that forms the blob. We have reviewed the observational evidence that support this idea: the acceleration pattern of the blobs, the V-shape that some of them exhibit, the collection of the material, the subsequent coherence of the blobs, and the observed retraction of the inner loop. Finally, we have suggested several observational tests for this theory. Numerical models should also be able to reproduce the proposed mechanism.

The authors thank S. Hill for his help in preparing the graphics, J. Kuijpers for many discussions and helpful comments, and the anonymous referee. M. K. van Aalst's work at the SOHO Experiment Analysis Facility at Goddard Space Flight Center was supported by ESA, the Olga Koningsfonds, the Leids Kerkhoven Bosscha Fonds, and Utrecht University. A. J. C. Beliën carried out this work on an ESA Fellowship. 


\section{REFERENCES}

Brueckner, G. E., et al. 1995, Sol. Phys., 162, 357

Dahlburg, R. B., Boncinelli, P., \& Einaudi, G. 1998, Phys. Plasmas, 5, 79

Einaudi, G., Boncinelli, P., Dahlburg, R. B., \& Karpen, J. T. 1998, J. Geophys. Res., in press

Gold, T. 1962, Space Sci. Rev., 1, 100

Gosling J. T. 1975, Rev. Geophys. Space Phys., 13, 1053

Gosling, J. T. 1993, J. Geophys. Res., 98, 18937

Gosling J. T. 1996, ARA\&A, 1996, 34, 35

Gosling, J. T., Birn, J., \& Hesse, M. 1995, Geophys. Res. Lett., 22, 869

Hiei, E., Hundhausen, A. J., \& Sime, D. G. 1993, Geophys. Res. Lett., 20, 2785

Hundhausen, A. J. 1993, J. Geophys. Res., 98, 13177

Hundhausen, A. J. 1998, in The Many Faces of the Sun, ed. K. Strong, J. Saba., \& B. Haisch (Berlin: Springer), in press

Illing, R. M. E., \& Hundhausen, A. J. 1983, J. Geophys. Res., 88, 10210

Kahler, S. W., \& Hundhausen, A. J. 1992, J. Geophys. Res., 97, 1619

Kopp, R. A. 1992, in Coronal Streamers, Coronal Loops, and Coronal and Solar Wind Composition, Proceedings of the First SOHO Workshop, ESA SP-348 (Noordwijk: ESA Publications Division), 53

Kopp, R. A., \& Pneuman, G. W. 1976, Sol. Phys., 50, 85

Kundu, M., Gopalswamy, S., White, P., Cargill, P., Schmahl, E. J., \& Hildner, E. 1989, ApJ, 347, 505

MacQueen, R. M. 1980, Philos. Trans. R. Soc. Lond. A, 297, 605

McComas, D. J., Gosling, J. T., \& Phillips, J. L. 1992, J. Geophys. Res., 97, 171

McComas, D. J., Gosling, J. T., Phillips, J. L., Bame, S. J., Luhmann, J. G., \& Smith, E. J. 1989, J. Geophys. Res., 94, 6907

McComas, D. J., Phillips, J. L., Hundhausen, A. J., \& Burkepile, J. T. 1991, Geophys. Res. Lett., 18, 73

Priest, E. R., et al. 1998, in A Crossroads for European Solar and Heliospheric Physics, ESA SP-417, ed. E. R. Priest, F. Moreno-Insertis, \& R. A. Harris (Noordwijk: ESA Publications Division), 204

Raymond, J. C., et al. 1997, Sol. Phys., 175, 645

Rust, D. M. 1997, BAAS, 29, 915

Sheeley, N. R., Jr., et al. 1997, ApJ, 484, 472

Wang, Y.-M., et al. 1998, ApJ, 498, L165

Webb, D. F., \& Cliver, E. W. 1995, J. Geophys. Res., 100, 5853

Wilcox, J. M. 1971, in Physics of the Solar Corona, ed. C. J. Macris (Dordrecht: Reidel), 88 